\title{
Antecedent chronic hyperglycaemia blocks phlorizin-induced insulin resistance in the dog
}

\author{
M. Koffler ${ }^{1}$, T. Imamura ${ }^{2}$, F. Santeusanio ${ }^{2}$ and J.H. Helderman ${ }^{3}$ \\ ${ }^{1}$ Center for Diabetes Research, ${ }^{2}$ Veterans Administration Hospital and \\ ${ }^{3}$ Department of Medicine, University of Texas Health Science Center at Dallas, Dallas, Texas, USA
}

\begin{abstract}
Summary. Hyperglycaemia may enhance insulin resistance typical of non-insulin dependent diabetes mellitus, as well as insulin dependent diabetes mellitus, and thus initiate a vicious pathogenetic cycle. We sought to test the hypothesis that reduction in chronic hyperglycaemia in the diabetic dog by methods that do not employ insulin may improve insulin resistance. We used the glucuretic agent phlorizin in dogs rendered chronically hyperglycaemic and diabetic by alloxan treatment. To analyse glucose disposition the euglycaemic clamp was performed. To minimize the role of counterregulatory influences that might be at play when glucose is reduced, the hyperglycaemic clamp with continuous somatostatin infusion was performed. Although phlorizin normalised
\end{abstract}

plasma glucose in the diabetic dog and reduced plasma glucose in normal, non-diabetic dogs, insulin dependent glucose disposition rate did not improve. While phlorizin itself was associated with insulin resistance in the normal animals, the insulin resistance of diabetes mellitus was not further augmented. We conclude that phlorizin is associated with insulin resistance perhaps by a common pathway shared by chronic hyperglycaemia. Care must be taken when phlorizin is used as an agent to study glucose disposition.

Key words: Insulin resistance, chronic hyperglycaemia, phlorizin, hyperinsulinaemic glucose clamps, somatostatin.
Resistance to insulin-mediated glucose uptake has been implicated as a factor in the pathogenesis of noninsulin-dependent diabetes mellitus [1-2]. It has been postulated that hyperglycaemia, which is a consequence of islet cell dysfunction and insulin resistance, is also an inducer of islet cell dysfunction and further insulin resistance [3]. If so, hyperglycaemia, once established, could initiate a vicious cycle of self-exacerbation which might explain the progressive nature of the metabolic derangements in diabetes mellitus.

Insulin resistance related to hyperglycaemia is thought to reside at a post-receptor locus often associated with glucose transport [4-6]. There is evidence that hyperglycaemia is associated with insulin resistance in alloxan diabetic dogs [7], in Type 1 (insulin-dependent) [8], and in Type 2 (non-insulin-dependent) [9] human diabetes. Chronic hyperglycaemia has been reported to reduce glucose transport sites in a variety of tissues [10-12]. In adipocytes hyperglycaemia-related insulin resistance is a consequence of depletion of glucose transporters that are otherwise recruited from an intracellular pool as a consequence of insulin action [12]. In obese diabetic patients, correction of hyperglycaemia for three weeks by multiple injections of insulin without changing body weight can eliminate the insulin resistance related to hyperglycaemia itself with- out altering residual weight-related insulin resistance [9].

If hyperglycaemia can produce a deleterious effect on glucose disposition, it is reasonable to postulate that maintenance of euglycaemia in diabetic patients or animals may significantly improve insulin sensitivity. The experiments reported here were designed to specifically test the hypothesis that chronic normalisation of blood glucose without the administration of insulin in diabetic dogs improved glucose sensitivity. The model that was chosen to create chronic normalisation of blood glucose in the dog employs the glucuretic agent, phlorizin. Phlorizin binds to the sodium-dependent active glucose transporter in the brush border of the proximal tubule of the kidney [13] thereby preventing glucose reabsorption; the resulting severe renal glucose "leak" normalises or significantly reduces glucose concentrations in the diabetic dog [14], and induces mild hypoglycaemia in normal dogs $[15,16]$.

\section{Materials and methods}

Severe insulin-requiring diabetes mellitus was induced in 9 normal mongrel male dogs weighing from $20-35 \mathrm{~kg}$ by a single intravenous injection of alloxan, $70 \mathrm{mg} / \mathrm{kg}$ body weight [17]. The dogs were maintained from the time when diabetes was induced (several days after injection) by careful attention to diet and insulin replacement 
for at least 3 months prior to study. The daily dose of insulin required to keep glycaemia of $9.5-12.8 \mathrm{mmol} / 1$ was $18-25$ units of isophane insulin (Eli Lilly, Inc., Indianapolis, Ind, USA) in two divided doses. During the whole of this period the dogs were clinically well, afebrile, with normal white blood cell count, apparently happy, eating well, vigorous and playful. Glucose disposition in the diabetic dogs was assessed by the glucose clamp technique of DeFronzo et al. [18]. Eight additional non-obese, non-diabetic dogs were maintained for use as control animals in the various experimental manoeuvers. The parameters of glucose homeostasis were measured during a series of glucose clamp experiments without phlorizin and $40 \mathrm{~h}$ after initiation of phlorizin injections. This timing was chosen based on previous experience in this laboratory [14] as the time necessary to normalise hyperglycaemia in the diabetic dog. Two grams of phlorizin (phloretin- $2^{\prime}-\beta$-D-glucoside, Sigma Chemical Co., St. Louis, Mo, USA) as a $40 \%$ solution in propylene glycol were administered subcutaneously on two consecutive days beginning $48 \mathrm{~h}$ after discontinuation of insulin therapy, followed by glucose clamping. To check the intrinsic effect of phlorizin, clamps were performed with and without phlorizin in normal dogs. A total of 32 separate clamps were performed in the 17 dogs. Pilot studies suggested the need for hyperglycaemic clamps, but to eliminate potential bias from a systematic sequence of studies, the clamps were performed in a random sequence. In each pair of dogs, those with and those without phlorizin, experiments in at least two dogs were performed as paired studies with the dogs serving as their own controls. No differences were observed in control or phlorizin studies when paired and unpaired studies were analysed, so the data were pooled.

Diabetic and normal dogs were studied employing two distinct glucose clamp protocols.

\section{Clamp studies}

After a 20 -h overnight fast and under pentothal and halothane anaesthesia, teflon catheters $(16 \mathrm{G})$ were inserted into a crural vein for somatostatin infusion and into a saphenous vein for infusion of insulin and 20\% D-glucose in water. A third catheter was inserted into a femoral artery for blood sampling. A urethral catheter was inserted for urine sampling. All urine was collected, the total volume for each time period was measured as was the glucose concentration. A Harvard pump (Harvard Instruments Co., Millis, Mass, USA model 2205 + speed controller model 552) was used for glucose infusion. For insulin infusion a Harvard pump (model 935) was used. Both protocols involved a primed, continuous insulin infusion at the rate of $40 \mathrm{mU} / \mathrm{min} / 1.73 \mathrm{~m}^{2}$ (regular iletin I, Eli Lilly \& Co.) to create steady-state hyperinsulinaemia. In one group of studies, animals were clamped at a euglycaemic level and in another series the animals were clamped at a hyperglycaemic level. In both sets of experiments somatostatin was infused at a rate of $5 \mu \mathrm{g} / \mathrm{min}$ to suppress the endogenous islet hormone output so as to avoid differences in endogenous insulin and glucagon levels in normal and diabetic animals during the clamp. The somatostatin infusate $(C M=9357-2 \mathrm{mg}$ batch RKM05, Clin-Midy) was prepared in isotonic saline at $5 \mu \mathrm{g}$ / $\mathrm{ml}$ with addition of $4 \mathrm{ml}$ of $\mathrm{dog}$ blood and $1 \mathrm{ml}$ trasylol (Aprotinin $10,000 \mathrm{KIU} / \mathrm{ml}$, Mobay Chemical Co., New York, NY, USA) per $250 \mathrm{ml}$ to prevent absorption to glass or plastic and proteolytic destruction. The target blood glucose in each of the clamp experiments was maintained with a coefficient of variation of less than $5 \%$ for the study in each $\operatorname{dog}$, by the use of a negative feedback algorithm in which glucose was measured at 2.5 -min intervals for the first $30 \mathrm{~min}$ and then at 5-min intervals for the remainder of a $2 \mathrm{~h}$ period using the technique of DeFronzo et al. [18]. Insulin and glucagon measurements were obtained at $10-\mathrm{min}$ intervals from $0-60 \mathrm{~min}$ and at 15-min intervals from $60-120 \mathrm{~min}$.

\section{Measurement of plasma glucose, immunoreactive insulin, immunoreactive glucagon and glucose utilisation}

The plasma glucose concentration during the clamp was measured using a glucose oxidase system by a Beckman glucose analyser (Beckman Instruments, Inc., Fullerton, Calif, USA). Immunoreactive insulin was measured by the Herbert modification [19] of the method of Yalow and Berson [20], immunoreactive glucagon by the method of Harris et al. [21], and free insulin was measured by a modification of the method of Kuzuya et al. [22].

The mean glucose utilisation (M) value for glucose metabolised was calculated as follows from the mean of three 20 -min periods from 60 to $120 \mathrm{~min}$ of the study: $\mathrm{M}\left(\mathrm{mg} \cdot \mathrm{min}^{-1} \cdot \mathrm{kg}^{-1}\right)=\mathrm{INF}-\mathrm{UC}$ $\mathrm{SC}$. [INF $=$ glucose infusion rate; $\mathrm{UC}=$ correction for urinary loss of glucose; $\mathrm{SC}=$ space correction, an adjustment for glucose that has either been added to or removed from the glucose space in a similar manner as DeFronzo et al. [18]. The space correction calculates that portion of the glucose infusion rate $\left(\mathrm{mg} \cdot \mathrm{min}^{-1} \cdot \mathrm{kg}^{-1}\right)$ that overfilled or underfilled the glucose space. The rate which overfilled the space is subtracted from the total infusion to provide the best estimate of total body glucose utilisation. The glucose concentration differences between the value measured at the beginning of the 20 -min time period and at its end was multiplied by a constant which converts $\mathrm{mg}$ / $\mathrm{dl}$ to $\mathrm{mg} / \mathrm{l}$, converts the $20 \mathrm{~min}$ time period to a rate per min and converts data as originally provided as $\mathrm{M}^{2}$ to body weight (BW), adjusts for blood water and the glucose space as follows:

$$
\begin{aligned}
& \mathrm{SC}=\left(\Delta \mathrm{G} \frac{\mathrm{mg}}{100 \mathrm{ml}}\right) \times\left(1000 \frac{\mathrm{ml}}{1}\right) \times \frac{(0.19)}{(0.94)} \times \frac{1}{20 \mathrm{~min}} \times 40 \mathrm{~L} \times(\mathrm{SA} \div \mathrm{BW}) \\
& \mathrm{SC}=(\Delta \mathrm{G})(4.043 \times \mathrm{SA} \div \mathrm{BW})
\end{aligned}
$$

The glucose space used in the equation is 0.19 of total body water [18] and 0.94 is taken as the fraction of a plasma specimen results in which glucose is diluted [18]. As hepatic glucose production is negligible during hyperinsulinaemia and at normal or higher plasma levels of glucose [23, 24], $M$ becomes a good estimate of the glucose utilisation rate.

\section{Statistical analysis}

All results are expressed as mean \pm standard error of the mean. Statistical analysis was performed with the paired and unpaired Student's t-test.

\section{Results}

\section{Effect of phlorizin on blood and urine glucose levels and basal glucagon levels}

Pretreatment with phlorizin decreased fasting plasma glucose significantly in the diabetic animals undergoing two different glucose clamp protocols. In the diabetic dogs undergoing the euglycaemic clamp protocol the glucose fell from $17.6 \pm 0.8$ to $5.7 \pm 0.2 \mathrm{mmol} / \mathrm{l}$ (mean \pm SEM) $(p<0.001)$. This significant impact of phlorizin on lowering the hyperglycaemia imposed by this model of diabetes in the dog was also seen in those animals studied with the hyperglycaemic clamp $(15.5 \pm 0.2$ to $4.0 \pm 0.3 \mathrm{mmol} / \mathrm{l})(p<0.001)$. Although less striking, phlorizin reduced fasting plasma glucose levels in the normal, non-diabetic dogs as well (4.5 \pm 0.2 to $4.2 \pm 0.3 \mathrm{mmol} / 1$ in the euglycemic clamp; $5.4 \pm$ 0.3 to $3.9 \pm 0.3$ in the hyperglycaemic clamp) (Table 1 ). The expected glucuretic response was achieved as there was a significant increase in total urine flow rate and urine glucose excretion rates after phlorizin treatment in both normal and diabetic animals in both clamp protocols (Table 1). The urinary glucose loss increased after phlorizin treatment from $0.3 \pm 0.1$ to 
Table 1. Physiologic parameters during glucose clamping as affected by phlorizin in normal and diabetic dogs

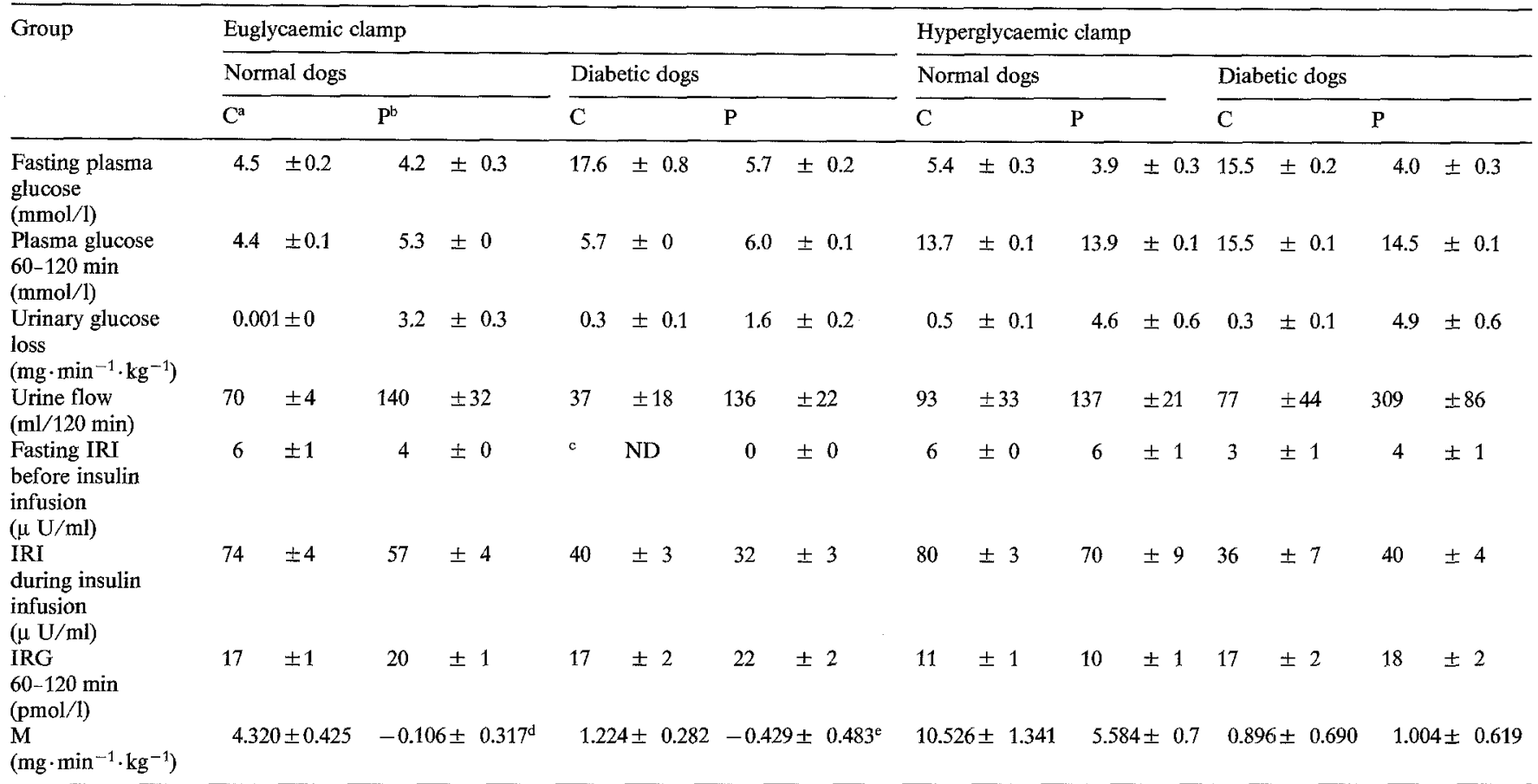

${ }^{a}$ Control without phlorizin; ${ }^{b}$ Experimental with phlorizin; ${ }^{c}$ Since insulin was infused to achieve normoglycaemia, the fasting IRI levels were irrelevant; ${ }^{d}$ A negative $\mathrm{M}$ occurs when urine losses are greater than glucose use by the cells $;{ }^{\mathrm{e}} \mathrm{ND}=$ Not measured. IRI $=$ Immunoreactive insulin; IRG $=\operatorname{Immunoreactive~ghicagon;~}$ $\mathrm{M}=$ Glucose utilisation rate

Table 2. Immunoreactive glucagon levels (pmol/1) before somatostatin, $15 \mathrm{~min}$ and $30 \mathrm{~min}$ after somatostatin infusion

\begin{tabular}{lrll}
\hline Groups of dogs & \multicolumn{3}{l}{ Time (min) } \\
\cline { 2 - 4 } & \multicolumn{1}{c}{0} & 15 & 30 \\
\hline Normal control & $30.2 \pm 4$ & $16.4 \pm 2$ & $15.0 \pm 2$ \\
Normal with P & $106.5 \pm 40$ & $25.5 \pm 7$ & $21.4 \pm 6$ \\
Diabetic control & $41.3 \pm 9$ & $26.7 \pm 3$ & $21.6 \pm 2$ \\
Diabetic with P & $209.5 \pm 37$ & $64.9 \pm 13$ & $34.9 \pm 5$ \\
\hline
\end{tabular}

$\mathbf{P}=$ phlorizin

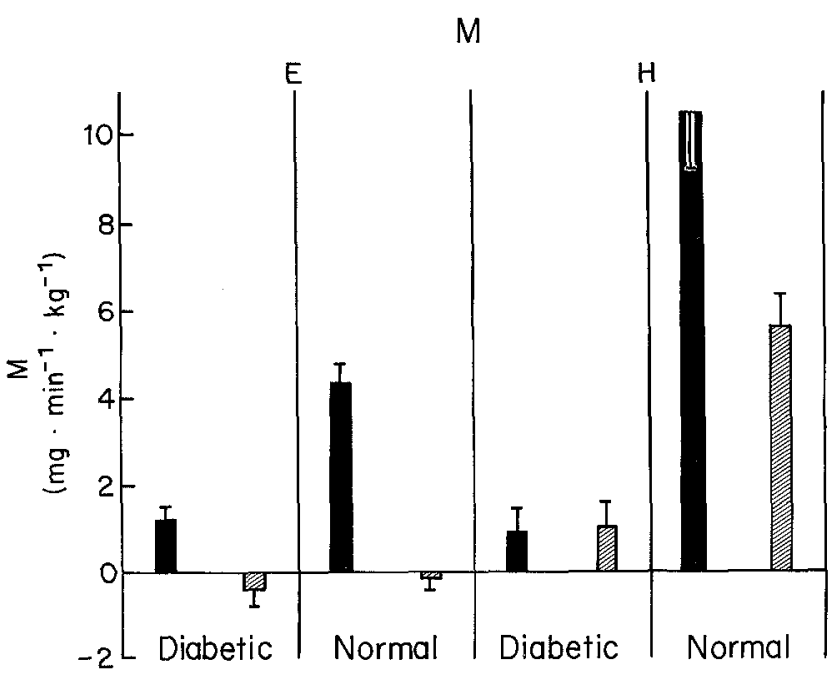

Fig. 1. Whole body glucose utilisation rate $\left(\mathrm{M} ; \mathrm{mg} \cdot \mathrm{kg}^{-1} \cdot \mathrm{min}^{-1}\right)$ in diabetic and normal dogs measured in the euglycaemic (E) and hyperglycaemic clamp $(\mathrm{H})$ as affected by phlorizin. $(\mathrm{D}=$ diabetic; $\mathrm{N}=$ normal; Black bars without phlorizin; hatched bars with phlorizin.)
$1.6 \pm 0.2 \mathrm{mg} \cdot \mathrm{min}^{-1} \cdot \mathrm{kg}^{-1}$ with an increase in flow rate from 37 to $136 \mathrm{ml} / 120 \mathrm{~min}$ in the diabetic dogs and from 0.001 to $3.2 \mathrm{mg} \cdot \mathrm{min}^{-1} \cdot \mathrm{kg}^{-1}$ in the normal animals when euglycaemia was maintained during the clamp (Table 1). In these normal dogs, phlorizin alone raised glucose urine loss from 0.001 to $3.9 \mathrm{mg} \cdot \mathrm{min}^{-1}$. $\mathrm{kg}^{-1}$ which fell to 3.2 after somatostatin was infused. Similarly, urine glucose increased from $0.3 \pm 0.1$ to $4.9 \pm 0.6$ in the diabetic dogs and from $0.5 \pm 0.1$ to $4.6 \pm 0.6$ in normal dogs when a hyperglycaemic stimulus was to be imposed during the clamp procedure. The difference in glycosuria between diabetic and normal dogs with phlorizin, somatostatin and euglycaemia is not readily apparent. Phlorizin not only reduced plasma glucose and increased urinary glucose excretion, it increased pre-clamp, pre-somatostatin immunoreactive glucagon from 30 to $106 \mathrm{pmol} / 1$ in normal dogs and from 41 to $210 \mathrm{pmol} / 1$ in the diabetic dogs (Table 2).

\section{Effect of phlorizin on glucose utilisation during the euglycaemic clamp}

Whole body glucose utilisation rate (the $M$ value) decreased markedly to "negative" values in both normal dogs and diabetic dogs during the euglycaemic clamp after pretreatment with phlorizin (Fig.1). Phlorizin did not compromise the capacity to achieve and maintain steady-state glucose during the clamp (Table 1). The steady-state plasma insulin concentrations achieved by the infusion of exogenous insulin were not statistically 
Table 3. The mean \pm SE of glucose infusion rate, urine loss, space correction and the glucose utilisation (M) value in normal and diabetic dogs with and without phlorizin during euglycaemic and hyperglycaemic clamps

\begin{tabular}{|c|c|c|c|c|c|c|}
\hline & & & $\begin{array}{l}\text { Infusion rate } \\
\left(\mathrm{mg} \cdot \mathrm{min}^{-1} \cdot \mathrm{kg}^{-1}\right)\end{array}$ & $\begin{array}{l}\text { Urine glucose loss } \\
\left(\mathrm{mg} \cdot \mathrm{min}^{-1} \cdot \mathrm{kg}^{-1}\right)\end{array}$ & Space correction & $\begin{array}{l}\mathrm{M} \\
\left(\mathrm{mg} \cdot \mathrm{min}^{-1} \cdot \mathrm{kg}^{-1}\right)\end{array}$ \\
\hline \multirow{4}{*}{$\begin{array}{l}\text { Euglycaemic } \\
\text { clamp }\end{array}$} & Normal & Control & $4.471 \pm 0.6$ & $0.001 \pm 0$ & $0.150 \pm 0.6$ & $4.320 \pm 0.425$ \\
\hline & dogs & Phlorizin & $3.647 \pm 0.2$ & $3.2 \pm 0.3$ & $0.567 \pm 0.2$ & $-0.106 \pm 0.317$ \\
\hline & Diabetic & Control & $1.423 \pm 0.3$ & \pm 0.1 & $-0.066 \pm 0.1$ & $1.224 \pm 0.282$ \\
\hline & dogs & Phlorizin & $1.565 \pm 0.4$ & $1.6 \pm 0.2$ & $0.031 \pm 0.3$ & $-0.423 \pm 0.483$ \\
\hline \multirow{4}{*}{$\begin{array}{l}\text { Hyperglycaemic } \\
\text { clamp }\end{array}$} & Normal & Control & $8.818 \pm 1.8$ & $0.5 \pm 0.1$ & $-0.395 \pm 0.9$ & $10.526 \pm 1.341$ \\
\hline & dogs & Phlorizin & $9.183 \pm 0.9$ & $4.6 \pm 0.6$ & $-0.025 \pm 0.7$ & $5.584 \pm 0.7$ \\
\hline & Diabetic & Control & $1.195 \pm 0.7$ & $0.3 \pm 0.1$ & $0.166 \pm 0.6$ & $0.896 \pm 0.690$ \\
\hline & $\operatorname{dogs}$ & Phlorizin & $4.819 \pm 1.1$ & \pm 0.6 & $-1.108 \pm 0.7$ & $1.004 \pm 0.619$ \\
\hline
\end{tabular}

affected by phlorizin, although in both groups there appeared to be a trend to lower basal insulin concentrations ( $20 \%$ on the average) after phlorizin administration. The means by which the $M$ was calculated in this study, using clamp glucose infusion rates, urinary glucose losses, and glucose space corrections, are displayed in Table 3. Negative $M$ values reflect large urinary losses. To test the impact of phlorizin on glucose homeostasis in the absence of chronic hyperglycaemia, euglycaemic clamps were performed in normal, nondiabetic animals. Phlorizin treatment was associated with a significant reduction $(p<0.001)$ in glucose utilisation in this group as well.

\section{Effect of phlorizin on glucose utilisation during hyperglycaemic clamp}

Because phlorizin-induced reduction of glucose concentrations might contribute to the unexpected insulin resistance in diabetic and non-diabetic dogs, animals were studied during a hyperglycaemic clamp. Steadystate plasma glucose concentrations were achieved during the clamp with a mean coefficient of variation of $4.3 \pm 0.1 \%$, for all clamps (Table 1) [18]. Again, there were no significant differences in the target glucose concentrations achieved before or after phlorizin in the diabetic dogs $(15 \pm 0.1$ vs $14.5 \pm 0.1 \mathrm{mmol} / \mathrm{l})$ or in the non-diabetic dogs $(13.7 \pm 0.1$ vs $13.9 \pm 0.1 \mathrm{mmol} / 1)$. Neither the plasma insulin concentrations achieved by exogenous infusion nor plasma glucagon levels measured were statistically altered by phlorizin treatment in the normal dogs undergoing the hyperglycaemic clamp. In these studies, steady-state insulin concentrations during the clamp were slightly reduced in the normal animals after phlorizin administration, a trend observed in both treatment groups during the euglycaemic clamp, although insulin concentrations achieved in the diabetic animals were slightly greater. As during the euglycaemic clamp, phlorizin treatment was associated with a significant reduction $(p<0.001)$ of glucose utilisation rate (M) in the non-diabetic dogs after phlorizin administration; however, there was no further reduction in glucose utilisation in the diabetic dogs. The M, far below that observed in the normal animals $\left(0.896 \pm 0.69\right.$ vs $\left.10.526 \pm 1.341 \mathrm{mg} \cdot \mathrm{min}^{-1} \cdot \mathrm{kg}^{-1}\right)$ was not further statistically reduced by phlorizin pretreatment (Table 1).

Somatostatin remarkably reduced the concentrations of glucagon during the $30 \mathrm{~min}$ before initiation of the clamp. Glucagon levels fell from $106 \mathrm{pmol} / 1$ before the initiation of the somatostatin infusion in the normal dog receiving phlorizin to $21 \mathrm{pmol} / \mathrm{l}$, a value not statistically different from the level after $30 \mathrm{~min}$ of somatostatin in the normal control that did not receive phlorizin (Table 3).

As there were no statistically significant changes in immunoreactive insulin (IRI) or immunoreactive glucagon (IRG) levels during the clamp in control or phlorizin treated animals, these hormones could not explain the observed changes of $M$ with phlorizin itself.

\section{Discussion}

Although these studies were designed to test the hypothesis that elimination of hyperglycaemia in diabetic dogs by means of an agent other than insulin would improve glucose disposition, pretreatment with a glucuretic agent, phlorizin, so markedly lowered the glucose disposition rate ( $\mathrm{M}$ value) - in normal and diabetic dogs during the euglycaemic clamp and in normal dogs during the hyperglycaemic clamp - that testing of the hypothesis was not possible. It is possible that the original hypothesis tested is wrong; that is to say, that rendering a chronically hyperglycaemic diabetic dog euglycaemic may not improve glucose tolerance. Several previous studies suggest that this explanation of the data is not correct [9, 14]. For example, Andrews et al. found in man that normalisation of blood glucose by insulin in hyperglycaemic obese Type 2 diabetic patients improved insulin sensitivity [9]. Furthermore, that phlorizin diminished glucose tolerance in normal, non-diabetic dogs whose baseline plasma glucose levels were not chronically elevated suggests that phlorizin itself has profound effects on glucose utilisation in dogs which obscure any potential improvement in glucose disposition that elimination of hyperglycaemia might have caused.

Although our understanding of the data compels us to conclude that phlorizin exerts a directly deleterious 
effect on glucose utilisation in these normal and diabetic dogs, three alternate explanations must be discussed. Firstly, it is possible that counterregulatory hormones (CRH) released in response to hypoglycaemia induced by phlorizin not suppressible by somatostatin infusions or hyperinsulinemia created during the clamp, could lead to a diminution in the $M$ values observed during the clamp. We believe, based on our reading of the applicable literature, that glucagon is the only relevant CRH in our model. Gauthier et al. [15] showed that intravenous phlorizin injection induced a reduction in plasma glucose levels of $15 \%$ in normal dogs. In that model of moderate hypoglycaemia, the compensatory increase in glucose production necessary to prevent severe hypoglycaemia in the face of the urinary glucose loss appeared to be brought about solely by an increase in the plasma glucagon concentration. In this study, they found no increase in plasma free fatty acid, thought by the authors to be a reliable index of the release of catecholamines, alternative candidates for potential CRH. In contrast to models of severe hypoglycaemia, Gauthier concluded that catecholamines do not play an important counterregulatory role in the defense of moderate hypoglycaemia. In a second study, the same authors [25] actually measured plasma levels of catecholamines, ACTH, and cortisol in normal dogs in the post-absorptive state after moderate hypoglycaemia was induced with phlorizin; they found no increases in CRH. Cryer [26] concluded that glucagon, by stimulating hepatic glucose production, plays a primary role in the defense of rapid decrements in plasma glucose levels. Epinephrine becomes critical mainly when glucagon is deficient or when hypoglycaemia is severe. Other hormones, neurotransmitters, and substrate effects are not sufficiently potent to prevent or correct moderate hypoglycaemia. Although there are differences between our study and the models used by Gauthier et al., there were data in our study to support a similar conclusion that glucagon could be the only CRH potentially defending phlorizin-induced hypoglycaemia. Since glucagon was inhibited by somatostatin, we feel that CRH cannot explain our findings. In the fasted state, plasma glucose is dependent on increased hepatic glucose production, presumably the result of stimulated $\mathrm{CRH}$, which, in the balanced state in which our dogs were studied, was associated with a degree of renal glucose leak caused by phlorizin $\left(3.928 \pm 0.5 \mathrm{mg} \cdot \mathrm{min}^{-1} \cdot \mathrm{kg}^{-1}\right)$. During the clamp, under the aegis of somatostatin which inhibits glucagon and growth hormone primarily, but not ACTH or catecholamines, we observed a significant fall in urinary glucose excretion $(3.221 \pm 0.5)$ which, because all of the parameters were held constant, could only have resulted from a reduction in hepatic glucose production. We conclude that inhibition of glucagon by somatostatin reversed the counterregulatory role of the liver under the influence of glucagon as proposed by Gauthier et al. and Cryer. To summarise our position on CRH, then, there are data to support the contention that glucagon was the only relevant $\mathrm{CRH}$ in the phlorizinised dog model which was removed from interpretative relevance during the clamp by somatostatin. Thus, we believe that the reduction in $M$ values caused by phlorizin during the clamp is best explained by a direct effect of the phlorizin molecule itself and not by the effects of potential CRH (which were irrelevant in this model).

A second potential explanation of the observation that glucose disposition in diabetic and in normal dogs under the influence of phlorizin was diminished, is the possibility that hepatic glucose production was not inhibited by hyperinsulinaemia achieved during the clamp, or by the somatostatin infusion-used specifically to have this physiologic effect. However, we do think that we were able to so diminish the impact of hepatic glucose gradient by the use of somatostatin as to negate this explanation. Our first contention is that hepatic glucose production is primarily driven by glucagon. Cherrington et al. [27] demonstrated that twothirds of basal glucose output of the liver of the dog is accounted for by glucagon. Furthermore, glucagon is the primary determinant of the blood glucose level in the post-absorptive state in the dog. Small increments in glucagon have a pronounced stimulatory effect on hepatic glucose output in the setting in which insulin concentration is fixed (such as during a euglycaemic clamp procedure) while hypoglucagonaemia with a fall in insulin will result in a significant fall in glucose production [28]. We observed a fall in urinary glucose excretion rate during the somatostatin infusion utilised in the clamp, suggesting a reduction in hepatic glucose production. Such a reduction would oppose the change in urinary glucose excretion (resulting from persistent or elevated hepatic glucose production) as a potential explanation for the fall of $\mathrm{M}$ observed in the phlorizinised animals. Furthermore, we took great pains to discuss the point that glucagon was the only relevant CRH potentially possible in our model, which we know is inhibited by somatostatin. We started the infusion of somatostatin $30 \mathrm{~min}$ before the clamp and continued the infusion for the duration of the study. We noted that somatostatin remarkably reduced the concentrations of glucagon during the $30 \mathrm{~min}$ before the clamp began (Table 2), a reduction which remained for the duration of the study (Table 1). Glucagon fell spectacularly from $106 \mathrm{pmol} / \mathrm{l}$ before the initiation of the somatostatin infusion in the normal animal receiving phlorizin to $21 \mathrm{pmol} / 1$, a value not statistically significantly different from the level after $30 \mathrm{~min}$ of somatostatin in the normal control dog that did not receive phlorizin. We observed an equally significant inhibition of glucagon by somatostatin $30 \mathrm{~min}$ after the infusion began, but before the clamp was started in the diabetic dogs with and without phlorizin (Table 2). As noted in Table 1, glucagon was further inhibited by the combination of somatostatin and hyperinsulinaemia 
effective during the clamp. These data, taken together with a reduction in urinary glucose loss during the clamp, strongly support the view that hepatic glucose production was inhibited by the inhibition of glucagon by somatostatin prior to the clamp; and further inhibited by the combination of hyperinsulinaemia and somatostatin inhibition of glucagon during the clamp. Thus, even without actual measurements of hepatic glucose production, it does not seem reasonable that failure of inhibition of hepatic glucose production can explain the significant reduction observed in $\mathbf{M}$ during the clamp induced by phlorizin. Again, we believe the best explanation of our data with respect to $M$ relates to a direct effect of phlorizin to reduce insulin responsiveness.

The final possible explanation of our data, other than the direct effect of phlorizin, relates to the degree of intrinsic insulin resistance established in the diabetic dog by the model of study chosen. If phlorizin has a positive effect on glucose homeostatis, then it is possible to argue that the severe diabetic state of the diabetic dogs could have masked this positive effect. Indeed, withdrawal of insulin for $88 \mathrm{~h}$ in the diabetic dogs prior to clamp study might have further increased the insulin resistance of that state of carbohydrate intolerance, ultimately accounting for the results relating to the $M$ during the clamp in the phlorizinised diabetic dogs. Unfortunately, this explanation of our data does not allow for the results of the phlorizinised normal dog since insulin and glucose were normal in that setting prior to study. The effect of phlorizin on glucose disposition during the clamp (M) is the same in the diabetic dogs and in the normal dogs; indeed, the effects were actually more severe in the normal animals than in the diabetic. It seems unreasonable to explain our data for only the diabetic groups which could not explain the more severe results in the normal animals. More reasonable to us would be a single, holistic explanation of these data, that is that phlorizin itself induced insulin resistance in our model. The concern about the degree of insulin resistance as measured by ketosis in the dog model is problematical. The dog, a carnivorous beast, is ketotic in the basal state. Ketosis, then, becomes a useless measure of the amount of insulin resistance in this model, the measure of which would not be helpful in the explanation of our data.

The mechanism by which phlorizin induces insulin resistance in the dog is not clear. It may be that phlorizin interferes with glucose homeostasis directly and/ or indirectly by virtue of stimulation of the release of several counterregulatory influences set in motion in response to the low-normal blood glucose levels encountered after phlorizin administration. Although cortisol, catecholamines and/or growth hormone were not measured, hypoglycaemia, the stimulus for release of these counterregulatory hormones, was eliminated as a confounding variable of interpretation of phlorizin effects on glucose disposition by performance of hy- perglycaemic clamps. These clamps, like the euglycaemic clamps, did not eliminate the effect of phlorizin pretreatment on the glucose disposition rate in the non-diabetic normal dogs, suggesting that this effect is not a consequence of counterregulatory influences resulting from hypoglycaemia. That the reduction in $\mathrm{M}$ was only $50 \%$ in the non-diabetic dog - far less than that observed during euglycaemia - suggests that any direct impact of phlorizin on glucose homeostasis may be limited or reduced by chronic hyperglycaemia that is imposed by the diabetic state. This potential interference in glucose utilisation induced by chronic hyperglycaemia is more striking in the diabetic animal with baseline insulin resistance and a low $\mathrm{M}$ value prior to phlorizin administration; phlorizin itself did not impose a further reduction in the $M$ values. This observation suggests that phlorizin, like chronic hyperglycaemia in the diabetic dog, can impair glucose disposition, perhaps at a common locus.

Plasma insulin levels were reduced in three of the four, but not all groups clamped after phlorizin administration. Although this trend did not reach statistical significance in any group, it may have contributed to the reduction in the $M$ values observed during the clamp. It may be that phlorizin may lead to enhanced renal handling of insulin either by direct enhancement of tubular handling or by increasing the filtered load of insulin. No reduction in steady-state insulin levels was greater than $20 \%$, yet the impairment in glucose disposition rate was many-fold greater than that supporting the view that phlorizin directly impairs glucose homeostasis. Plasma glucagon levels were suppressed by somatostatin during the clamps (Table 1) and certainly would not explain the changes observed in glucose disposition after phlorizin pretreatment.

Recently, Rossetti et al. [29] reported that phlorizin could normalise insulin sensitivity in mildly diabetic rats without chronic fasting hyperglycaemia. They found no deleterious effect of phlorizin on glucose homeostasis in the non-diabetic, normal rat. The differences between the findings of Rossetti et al. and our own may reflect differences in the severity of diabetes imposed on the animal models. The alloxan-treated dogs that we used were severely diabetic. In contrast, the partially pancreatectomised rats of Rossetti et al. were models of very mild diabetes with normal fasting glucose and insulin values when studied. In the absence of chronic hyperglycaemia and altered basal insulin levels, baseline glucose disposition rates in these "diabetic" animals were not abnormal and therefore, did not constitute a true test of the original hypothesis concerning the role of chronic hyperglycaemia in glucose homeostasis. It is possible, then, that phlorizin has a different impact on severely diabetic animals not discerned in the mildly affected. In any case, our findings, vis-a-vis the studies in the rat, suggest that care must be taken when choosing the model of study for further in- 
vestigation into the role of phlorizin on glucose disposition.

In conclusion, phlorizin profoundly reduces glucose utilisation itself in diabetic dogs. That phlorizin pretreatment failed to worsen insulin resistance observed in the hyperglycaemic clamp in the face of previous chronic hyperglycaemia of the diabetic dog suggests that factors responsible for the phlorizin effect may be suppressed by chronic hyperglycaemia, perhaps because the defect shares common pathways. One should be cautious about the use of the phlorizin model in explanation of the impact of hyperglycaemia on glucose disposition.

Acknowledgements. These studies were done under the sponsorship of NIH grants AM22150, and AMO2700-25 and the VA Institutional Research Support Grant 549-8000-01. Dr. Koffler is a recipient of a fellowship from the American Physicians Fellowship and the Upjohn Young Investigator Award. The authors wish to thank the following persons for their expert technical assistance: Ms. H.Gibson, Ms. V.Harris, Ms. K.McCorkle, Mr. W.McFarland, Ms. L.Peace, and Mr. D. Sandlin; and Ms. L. Lee for secretarial assistance.

\section{References}

1. Shen SW, Reaven GM, Farquhar JW (1970) Comparison of impedance to insulin-mediated glucose uptake in normal subjects and in subjects with latent diabetes. J Clin Invest 49:2151-2160

2. Reaven GM, Bernstein R, Davis B, Olefsky JM (1976) Nonketotic diabetes mellitus: insulin deficiency or insulin resistance? Am J Med 60: 80-88

3. Unger RH, Grundy S (1985) Hyperglycemia as an inducer as well as a consequence of impaired islet cell function and insulin resistance: implications for the management of diabetes. Diabetologia 28: 119-121

4. Howard BV, Savage PJ, Nagulesparan M, Benion LJ, Unger RH, Bennett PH (1979) Evidence for marked sensitivity to the antilipolytic action of insulin in obese maturity-onset diabetics. Metabolism 28: 744-750

5. Klimes I, Nagulesparan M, Vasquez V, Hidaka $H$, Unger RH (1984) Normal insulin sensitivity of the islets of Langerhans in obese subjects with resistance to its glucoregulatory actions. Diabetes 33: 305-310

6. Kolterman OG, Insel J, Saekow M, Olefsky JM (1980) Mechanisms of insulin resistance in human obesity. Evidence for receptor and postreceptor defects. J Clin Invest 65: 1272-1284

7. Reaven GM, Sageman WS, Swenson RS (1977) Development of insulin resistance in normal dogs following alloxan-induced insulin deficiency. Diabetologia 13: 459-462

8. DeFronzo RA, Hendler R, Simonson D (1982) Insulin resistance is a prominent feature of insulin-dependent diabetes. Diabetes 31: 795-801

9. Andrews WJ, Vasquez B, Nagulesparan M, Klimes I, Foley J, Unger RH, Reaven GM (1984) Insulin therapy in obese noninsulin dependent diabetes induces improvements in insulin action and secretion which are maintained for two weeks after insulin withdrawal. Diabetes 33: 634-642

10. Gjedde A, Crone C (1981) Blood-brain glucose transfer: depression in chronic hyperglycemia. Science $214: 456-457$

11. McCall AL, Millington WR, Wurtman RJ (1982) Metabolic fuel and amino acid transport into the brain in experimental diabetes mellitus. Proc Natl Acad Sci 79: 5406-5410
12. Karnieli E, Hissin PJ, Simpson IA, Solans LB (1981) A possible mechanism of insulin resistance in the rat adipose cell in streptozotocin-induced diabetes mellitus. Depletion of intracellular glucose transport system. J Clin Invest 68: 111-114

13. Silverman M (1981) Glucose reabsorption in the kidney. Can $\mathbf{J}$ Physiol Pharmacol 59: 209-224

14. Starke A, Grundy S, McGarry JD, Unger RH (1985) Correction of hyperglycemia with phlorizin restores the glucagon response to glucose in insulin-deficient dogs: implications for human diabetes. Proc Natl Acad Sci 82: 1544-1546

15. Gauthier C, Vranic M, Hetenyi Jr G (1980) Importance of glucagon in regulatory rather than emergency responses to hypoglycemia. Am J Physiol 238: E131-E140

16. Gauthier C, Hetenyi G (1982) Origin of glucose released in the regulatory response against hypoglycemia. Metabolism 31: $147-153$

17. Kawai K, Unger RH (1983) Opposing action of glucagon and insulin on splanchnic D cell function. J Clin Invest 71: 721-725

18. DeFronzo RA, Tobin JD, Andres R (1979) Glucose clamp technique: a method for quantifying insulin secretion and resistance. Am J Physiol 237: E214-E223

19. Herbert V, Lau KS, Gottlieb CW, Bleicher SJ (1965) Coated charcoal immunoassay of insulin. J Clin Endocrinol Metab 25: 1375-1384

20. Yalow RS, Berson SA (1960) Immunoassay of endogenous plasma insulin in man. J Clin Invest 39: 1157-1175

21. Harris V, Faloona GR, Unger RH (1979) Glucagon. In: Jaffe BM, Behrman HR (eds) Methods of hormone radioimmunoassay. Academic Press, New York, pp 643-656

22. Kuzuya H, Blix PM, Horwitz DL, Steiner DF, Rubenstein AH (1977) Determination of free and total insulin and c-peptide in insulin-treated diabetics. Diabetes 26: 22-29

23. Bondy PK, Bloom WL, Whitner VS, Farrar BW (1949) Studies of the role of the liver in human carbohydrate metabolism by the venous catheter technique. II. Patients with diabetic ketosis, before and after the administration of insulin. J Clin Invest 28 : 1126-1133

24. Rizza RA, Mandarino LJ, Gerich JE (1981) Dose-response characteristics for effects of insulin on production and utilization of glucose in man. Am J Physiol 240: E630-E639

25. Gauthier C, Vranic M, Hetenyi Jr G (1983) Nonhypoglycemic glucoregulation: role of glycerol and glucoregulatory hormones. Am J Physiol 244: E373-E379

26. Cryer PE (1985) Does central nervous system adaption to antecedent glycemia occur in patients with insulin-dependent diabetes mellitus. Ann Intern Med 103: 284-286

27. Cherrington AD, Lacy WW, Chiasson JL (1978) Effect of glucagon on glucose production during insulin deficiency in the dog. $J$ Clin Invest 62: 664-677

28. Unger RH, Orci L (1983) Glucagon. In: Ellenberg M, Rifkin H (eds) Diabetes mellitus. Medical Examination Publishing, New York, pp 203-224

29. Rossetti L, Smith D, Shulman GI, Papachristou D, DeFronzo RA (1987) Correction of hyperglycemia with phlorizin normalizes tissue sensitivity to insulin in diabetic rats. J Clin Invest 79: 1510-1515

Received: 16 June 1987

and in revised form: 2 February 1988

Dr. J. Harold Helderman

Department of Medicine

University of Texas

Southwestern Medical Center

5323 Harry Hines Blvd.

Dallas, Texas 75235-9030

USA 